\title{
Operation of probe heads on the Multi-Purpose-Manipulator at W7-X in OP 1.2a
}

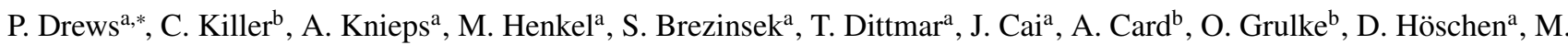 \\ Jakubowski ${ }^{\mathrm{b}}$, R. König ${ }^{\mathrm{b}}$, A. Krämer-Flecken ${ }^{\mathrm{a}}$, Y. Liang ${ }^{\mathrm{a}}$, Y. Li ${ }^{\mathrm{a}}$, C. Linsmeier ${ }^{\mathrm{a}}$, S. Liu ${ }^{\mathrm{a}, \mathrm{c}}$, D. Nicolai ${ }^{\mathrm{a}}$, O. Neubauer ${ }^{\mathrm{a}}, \mathrm{G}$ \\ Satheeswaran $^{\mathrm{a}}$, N. Sandri ${ }^{\mathrm{a}}$, B. Schweer ${ }^{\mathrm{a}}$, T. Schröder ${ }^{\mathrm{b}}$, and the W7-X Team ${ }^{\mathrm{b}}$ \\ ${ }^{a}$ Forschungszentrum Jülich GmbH, Institut für Energie- und Klimaforschung Plasmaphysik, \\ Partner of the Trilateral Euregio Cluster (TEC), 52425 Jülich, Germany \\ ${ }^{b}$ Max-Planck-Institut für Plasmaphysik,17491 Greifswald, Germany \\ ${ }^{c}$ Institute of Plasma Physics, Chinese Academy of Sciences, Hefei 230031, People's Republic of China
}

\begin{abstract}
The Multi-Purpose-Manipulator (MPM) has been operated, as a versatile carrier system for probes, since the first campaign OP. 1.1 in 2015 at Wendelstein 7X. The MPM is mounted at the outboard midplane and is able to perform fast plunges through the entire scrape off layer up to the last closed flux surface. In addition, a system for gas injection was commissioned that allowed the use of a dedicated probe head and a port on the existing probes for fueling and impurity seeding experiments. A combined probe, a combination of Langmuir, Mach and magnetic probes, was used. For the second campaign OP. 1.2a in 2017, with an island divertor, an upgraded combined probe, a fluctuation probe, a retarding field analyzer, a Mach probe array, a laser blow off target and a material probe holder were added to the range of plasma edge diagnostics. The new diagnostic probe heads were designed to address specific tasks, such as measuring the edge fluctuations, the ion temperature and the radial Mach number profiles. However, all fast plunging probes were designed such that they provide the most important quantities for SOL characterization (electron temperature, density, radial electric field).
\end{abstract}

Keywords: W7-X,Multi-purpose Manipulator, Langmuir probes, plasma edge transport

\section{Introduction}

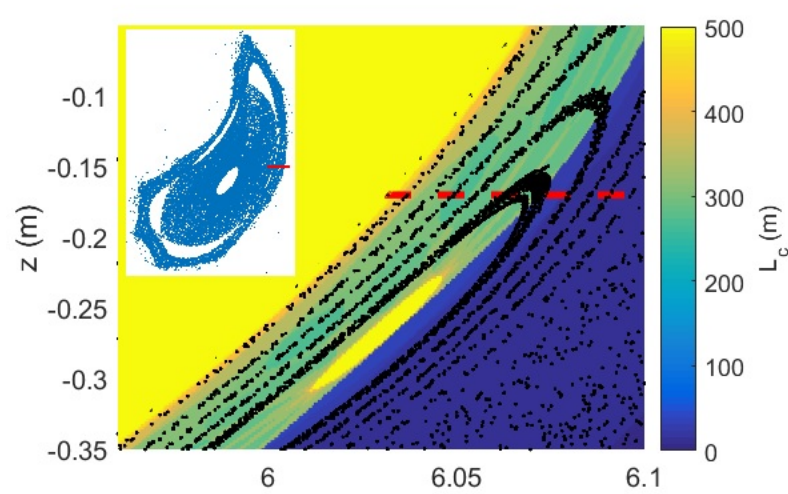

Figure 1: Poincare plot of the magnetic island probed by the MPM in the toroidal plane $p h i=200.7^{\circ}$ for the $\mathrm{W} 7-\mathrm{X}$ magnetic standard configuration (black dots) combined with the colorcoded connection length. Upper left corner: full view of the full poincare cross section

The Multi-purpose Manipulator was installed at W7-X in 2015 and is in operation since the first experimental phase OP1.1 (2015/16) [1] [2]. The MPM is able to reach the last closed flux surface in all magnetic configuration. On this way,

\footnotetext{
${ }^{*}$ Corresponding author

Email address: p.drews@fz-juelich.de (P. Drews )
}

it crosses the intrinsic magnetic island chain which is pivotal for the island divertor employed at W7-X, as indicated in figure 1. The plunge depth of the manipulator has to be adjusted depending on the configuration, the applied heating power, the planned density and the expected bootstrap current. For measurements with the MPM a variety of probes and material holders were developed. The comparison of their capabilities are detailed in table 1 . The probes considered for this report shown in figure 2 are the combined probe (FZJ-COMB2), fluctuation probe (IPP-FLUC1) and the RFA (Retarding Field Analyzer) probe (FZJ-RFA1, FZJ-RFA2)). The combined probe is an upgrade from a previous model from the OP. 1.1 [3] experiments. The probe head has a set of triple Langmuir pins for the measurement of the electron temperature $T_{\mathrm{e}}$ and density $n_{\mathrm{e}}$, poloidally arrayed floating potential pins, a Mach probe setup, an ion sensitive probe (ISP) and a 3D pick up coil. The guard ring of the ion senstive probe functions like a swept Langmuir probe.

The fluctuation probe features a four pin mach probe and 22 Langmuir pins poloidally arrayed in two lines, those pins were selected to be operated as floating potential pins and ion saturation pins, with one on a swept biasing for electron temperature and density measurements. From the poloidal array the poloidal propagation velocity of turbulent fluctuations can be estimated by correlation analysis, allowing to deduce the radial electric field under the assumption the poloidal 


\begin{tabular}{|l|l|l|l|l|}
\hline Probe & $T_{\text {eTriple }}$ & $T_{\text {eS wept }}$ & $T_{\mathrm{i}}$ & Mach \\
\hline $\begin{array}{l}\text { FZJ- } \\
\text { COMB2 }\end{array}$ & Yes & $\begin{array}{l}\text { ISP } \\
\text { Guard }\end{array}$ & ISP & Yes \\
\hline $\begin{array}{l}\text { IPP- } \\
\text { FLUC1 }\end{array}$ & Yes & Yes & n/a & $\begin{array}{l}\text { Four- } \\
\text { directional }\end{array}$ \\
\hline $\begin{array}{l}\text { FZJ- } \\
\text { RFA1 }\end{array}$ & n/a & Yes & Yes & n/a \\
\hline $\begin{array}{l}\text { FZJ- } \\
\text { RFA2 }\end{array}$ & Yes & Yes & Yes & n/a \\
\hline
\end{tabular}

Table 1: Capabilities of the probes heads used in OP 1.2a

dynamics are $\vec{E} \times \vec{B}$ driven. The installed four pin Mach probe [4] allows to deduce both parallel and poloidal Mach numbers.

An RFA probe was developed primarily to measure the ion temperature profiles $T_{\mathrm{i}}$ in the plasma edge [5] of OP 1.2. It also posseses two Langmuir pins on the plasma facing top side of the probe head, one operated on a floating potential and the other one with a swept biasing. The second iteration of the RFA probe for OP $1.2 \mathrm{~b}$ was modified to accomodate two extra Langmuir pins for a Triple Probe setup. Furthermore a head
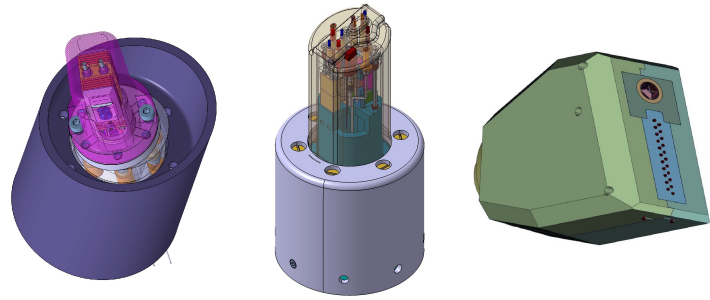

Figure 2: Left: Retarding field analyzer probe (FZJ-RFA1), middle: combined probe (FZJ-COMB2), right: fluctuation probe (IPP-FLUC1)

for a Laser Blow Off (LBO) system [6] and a material sample holder were deployed in OP 1.2a.

\section{Gas puffing}

The probes have al been fitted with a gas puffing port to allow gas puffing from the manipulator system. In OP 1.A a basic scheme for gas puffing through the MPM was available: To trigger the puffing the pneumatic valve which decouples the MPM gas reservoir from the moving manipulator parts was opened for a short time (typically 250 to $500 \mathrm{~ms}$ ) and the total amount of puffed gas was controlled by filling the gas reservoir of the MPM gas handling system to different pressures. The gas was allowed to free stream along the feed lines of the MPM (ca. $6 \mathrm{~m}$ length and $6 \mathrm{~mm}$ tubing) and as injected by a small metal capillary in the probe head (usually $4 \mathrm{~mm}$ inner diameter). Linearity of the total amount of gas dosed versus reservoir pressure was verified by tests in the MPM exchange chamber by comparing the pressure drop in the gas reservoir. Total amount of up to 140 mbarl seeding per discharge were realized in OP 1.2a. Detailed data on peak flow rate or the time variation of the flow are not available but the injection was usually visible by camera observations of the injection plume with the background plasma for a few seconds. For OP $1.2 \mathrm{~b}$ the same system will be used in the existing probe heads, but an additional probe head for controlled gas puffing employing and internal reservoir and a piezo valve directly in the head are in preparation.

\section{Conditioning of the probes}

An important lesson for the operation of probes was that sufficient baking with both graphite and boron-nitride covers is necessary. All probes were baked at a minimum of $130{ }^{\circ} \mathrm{C}$ to achieve about $10^{-5}$ mbar vacuum. This relativley low temperature was decided to protect plastic components at the probe interface and cable isolation in the probe itself. In OP $1.2 \mathrm{a}$ an arcing event caused a cable to be point-welded unto the stainless steel superstructure and burned a hole into the RFA grid. This arcing is possibly caused by outgassing which will happen during plasma exposure. A possible solution, until baking of probes in the exchange chamber of the MPM is available, is the exposure of the probes to the plasma, without biasing or lower voltages, for the first few discharges. This is not a full garantue that further outgassing will not occur if the probe is sufficiently heated by the plasma. For this issue the current that flows from the pins or the RFA grids is limited by installing an additional resistor of $1 \mathrm{k} \Omega$ to protects the RFA grids for OP $1.2 \mathrm{~b}$.

\section{Probe measurements}

The floating potentials of up to $300 \mathrm{~V}$ in OP $1.2 \mathrm{a}$ by far exceeded the expectation from the measurements in OP 1.1. They are generally asociated with the plasma density, normally the higher the density the lower are the floating potentials. The triple probe utilized two supercap modules that could supply combined up to $300 \mathrm{~V}$ with $10 \mathrm{~A}$ while the Kepco amplifier could only supply $\pm 200 \mathrm{~V}$ with up to $1 \mathrm{~A}$ current. For OP $1.2 \mathrm{~b}$ an additional Dana amplifier with a biasing of $\pm 300 \mathrm{~V}$ and a current of $2 \mathrm{~A}$ was installed. An additional off-set from the supercap modules for the swept biasing was introduced for the measurements with the RFA probe. The mach probes measurements used a single supercap module with $150 \mathrm{~V}$ of biasing. The swept biasing considering the long transmission of about $12 \mathrm{~m}$ has considerable capacity effects and while filtering is possible, only a slow sweeping of about $1 \mathrm{kHz}$ is advised. The amplifier based biasing system also were more likely to experience a current limit and therefore a biasing fluctuation. Figure 3 shows the biasing voltage, the total current of the guard ring together with the correction for the capacity effect and the true collection current signal. A fourier technique [7] was used to correct the capacity effects that are present in the electronics. The originally collected current appears to have a phaseshift to the biasing voltage by about $90^{\circ}$. To obtain the background component, the transfer function of the probe system is estimated from the signals in-between the plunges and then applied to the bias curve during the plunge. This method is able to recover the signal necessary to apply the characteristic $U-I$ curve 


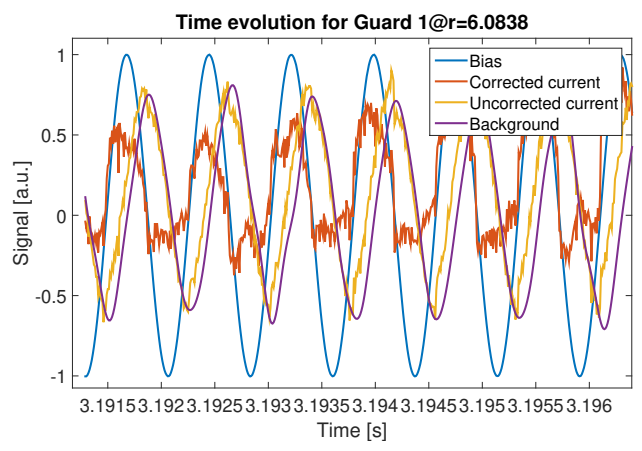

Figure 3: Unfiltered collection current of the swept Langmuir pin (Ion sensitiy probe, of the combined probe, middle: fourier filtered collection current, bottom: Biasing voltage

for Langmuir probes to obtain the electron temperature. The swept probes while being limited by capacity effects have the obvious advantage that their measurement is not depending on a combination of Langmuir pins functioning at the same time. In figure 4 the measurements from the RFA probe from OP $1.2 \mathrm{~b}$ are shown, the expected voltage to current curve $U-I$ with ion collection at negative voltages and the repelling of electrons for positive voltages is recorded. The RFA probe is also sus-

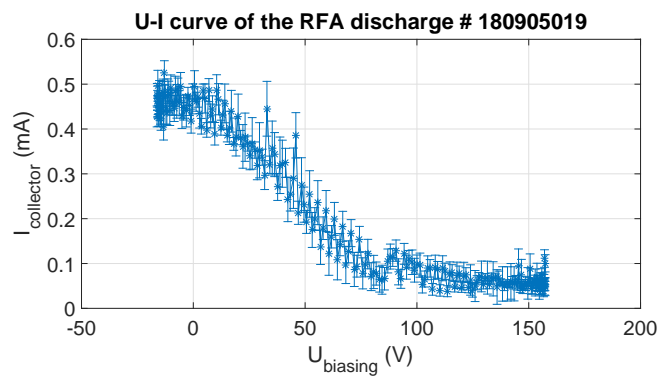

Figure 4: Characteristic voltage-current curve of the RFA probe

ceptible to capacity effects due to the close proximity of swept voltages on the grids and the use of ceramics that are inherently dielectric. While the dielectric effects can be avoided, by choosing aluminum-oxide with a low dielectric constant, the tightly packed grid structure is inherent. Thus rather low frequencies for the sweeping voltages of about $1 \mathrm{kHz}$ are employed. The temporal resolution of the swept Langmuir probe with $1 \mathrm{kHz}$ is rather low. The profiles of the electron temperature from the RFA and combined probe are able to resolve the local peaking in the vicinity of the island remnant. Figure 5 shows the measurements of the combined probe with both a triple probe and a swept Langmuir probe in the OP 1.2 standard configuration. The temporal resolution of the swept Langmuir probe with $1 \mathrm{kHz}$ is rather low, but the swept probe resolves a profile similar to the triple probe. The error of the swept probe was calculated from the fitting error of a $85 \%$ confidence bounds. The guard ring used as a swept Langmuir pin, is a hollow tungsten cylinder which has considerable consequence on the saturation currents, this is affecting the measured electron density and not the temperature. A higher sweeping frequencies for a higher temporal and spatial resultion of the temperature and density

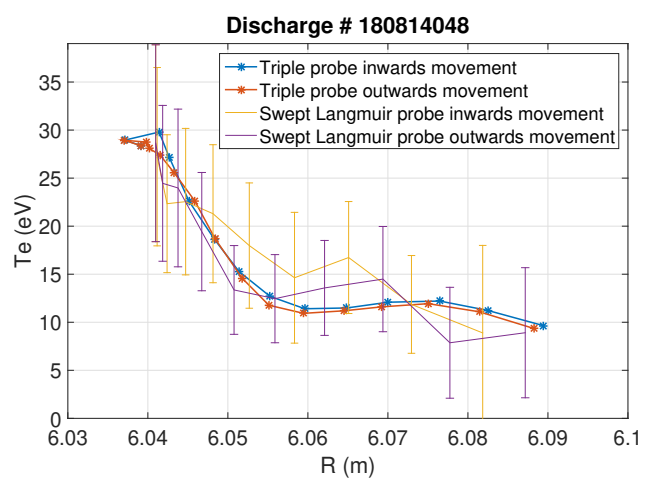

Figure 5: Measured electron temperature profiles from the combined probe using both the triple and single swept Langmuir probe

profiles is needed.

\section{Conclusion}

The three probes are dedicated to different measurements, still the electron temperature and density and deduced quantities like the electric field can be measured by all. Having both swept and constant biased Langmuir probes has therefore the benefit of electron temperature and density measurements at high temporal resolution and technical reliability. Certain functionalitites like the capability to measure the ion temperature, a 3D pick-up coil and a poloidal array of Langmuir pins should be included in a future probe head for OP 2 in addition.

\section{Acknowledgements}

This work has been carried out within the framework of the EUROfusion Consortium and has received funding from the Euratom research and training program 2014-2018 under grant agreement No 633053. The views and opinions expressed herein do not necessarily reflect those of the European Commission.

\section{References}

[1] G. Satheeswaran, A pcs7-based control and safety system for operation of the w7-x multi-purpose manipulator facility, Fusion Engineering and Design $123 \quad$ (2017) 699-702 (2017). doi:https://doi.org/10.1016/j.fusengdes.2017.05.125.

[2] D. Nicolai, A multi-purpose manipulator system for w7-x as user facility for plasma edge investigation, Fusion Engineering and Design 123 (2017) 960-964 (2017). doi:https://doi.org/10.1016/j.fusengdes.2017.03.013.

[3] P. Drews, Measurement of the plasma edge profiles using the combined probe on w7-x, Nuclear Fusion 57 (2017) 126020 (2017). doi:https://doi.org/10.1088/1741-4326/aa8385.

[4] N. Smick, Wall scanning probe for high-field side plasma measurements on alcator c-mod, Review of Scientific Instruments 80 (2009) 023502 (2009). doi:https://doi.org/10.1063/1.3069290.

[5] M. Henkel, Multi-channel retarding field analyzer for east, Plasma Sci. Technol. 20 (2018) 054001 (2018). doi:https://doi.org/10.1088/2058$6272 / \mathrm{aab} 490$

[6] T. Wegner, Design, capabilities, and first results of the new laser blowoffsystem on wendelstein 7-x, Review of Scientific Instruments 89 (2018) 073505 (2018). doi:https://doi.org/10.1063/1.5037543. 
[7] D. Brunner, Scanning ion sensitive probe for plasma profile measurements in the boundary of the alcator c-mod tokamak, Review of Scientific Instruments 84 (2013) 053507 (2013). doi:https://doi.org/10.1063/1.4807699. 